\title{
A MUSE inquiry into the physical processes taking place within the Abell 2667 Brightest Cluster Galaxy
}

E. Iani ${ }^{1,2}$ D, G. Rodighiero ${ }^{1,3}$, J. Fritz ${ }^{4}$, G. Cresci ${ }^{5}$, C. Mancini $^{1,3}$, P. Tozzi ${ }^{5}$, L. Rodríguez-Muñoz ${ }^{1}$, P. Rosati ${ }^{6}$, G. B. Caminha ${ }^{7}$,

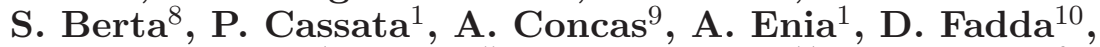
A. Franceschini ${ }^{1}$, A. Liu ${ }^{5}$, A. Mercurio ${ }^{11}$, L. Morselli ${ }^{9}$, P. G. Pérez-González ${ }^{12,13}$, P. Popesso ${ }^{9}$, G. Sabatini $^{14,15}$ and A. Zanella ${ }^{2}$

${ }^{1}$ Dipartimento di Fisica ed Astronomia, Università degli Studi di Padova, Vicolo dell'Osservatorio 3, I-35122 Padova, Italy email: edoardo.iani@phd.unipd.it

${ }^{2}$ European Southern Observatory, Karl Schwarzschild Straße 2, D-85748 Garching, Germany ${ }^{3}$ INAF - Osservatorio Astronomico di Padova, Vicolo dell'Osservatorio 5, I-35122 Padova, Italy

${ }^{4}$ Instituto de Radioastronomía y Astrofísica, UNAM, Campus Morelia, A.P. 3-72, C.P. 58089, Mexico

${ }^{5}$ INAF - Osservatorio Astrofisico di Arcetri, Largo Enrico Fermi 5, I-50125 Firenze, Italy

${ }^{6}$ Dipartimento di Fisica e Scienze della Terra, Università degli Studi di Ferrara, Via Saragat 1, I-44122 Ferrara, Italy

${ }^{7}$ Kapteyn Astronomical Institute, University of Groningen, Postbus 800, 9700 AV Groningen, The Netherland

${ }^{8}$ Institut de Radioastronomie Millimétrique 300 rue de la Piscine, Domaine Universitaire 38406 Saint Martin d'Hères, France

${ }^{9}$ Excellence Cluster Universe, Boltzmannstraße 2, D-85748 Garching, Germany ${ }^{10}$ SOFIA Science Center, USRA, NASA Ames Research Center, M.S. N232-12 Moffett Field, CA 9403

${ }^{11}$ INAF - Osservatorio Astronomico di Capodimonte, via Moiariello 16, 80131 Napoli, Italy

${ }^{12}$ Departamento de Astronomía y Astrofísica, Universidad Complutense de Madrid, Av. Complutense s/n, C.P. 28040, Madrid, Spain

${ }^{13}$ Centro de Astrobiología (CAB, INTA-CSIC), Carretera de Ajalvir km 4, E-28850 Torrejón de Ardoz, Madrid, Spain

${ }^{14}$ Dipartimento di Fisica e Astronomia, Università degli Studi di Bologna, via Gobetti 93/2, I-40129 Bologna, Italy

${ }^{15}$ INAF - Istituto di Radioastronomia - Italian node of the ALMA Regional Centre (ARC), via Gobetti 101, I-40129 Bologna, Italy

\begin{abstract}
Brightest cluster galaxies (BCGs) residing in cool-core clusters are known to be the stage of intricate baryon cycle phenomena (e.g. gas inflows, AGN outflows, star formation feedback). The scenarios describing the observed properties of these galaxies are still controversial, suffering from limitations due to the spatial resolving power of the instruments, specifically for galaxies beyond the Local Universe. However, the dramatic improvements introduced by the integral-field unit instruments (e.g. MUSE) could shed light on the physical processes driving the evolution of these galaxies. We present an extensive analysis of the stellar and gas properties (i.e. kinematics, stellar mass, star formation rate) of the radio-loud BCG sitting at the centre of the X-ray luminous cool-core cluster Abell $2667(z=0.23)$, based on MUSE data. Our
\end{abstract}


results indicate that the BCG is a massive elliptical, hosting an AGN that is possibly undergoing accretion of cold star-forming clouds of ICM or galactic cannibalism.

Keywords. galaxies: elliptical and lenticular, cD; galaxies: evolution; galaxies: kinematics and dynamics; galaxies: nuclei; (galaxies:) cooling flows

\section{Introduction}

Brightest cluster galaxies (BCGs) are the largest, most massive and luminous galaxies in the Universe. They host relatively old stellar populations and little on-going star formation activity, thus representing the brightest end of the luminosity function of earlytype galaxies (ETGs). In dynamically relaxed galaxy clusters (i.e. no recent mergers with nearby clusters), BCGs sit almost at rest at the bottom of clusters potential wells and close to the peak of the thermal X-ray emission originated by the hydrostatical cooling of the hot $\left(10^{7}-10^{8} \mathrm{~K}\right)$ intracluster medium (ICM).

Despite their classification as ETGs, if specific environmental conditions are met, BCGs have been found to show exceptional properties over the whole electromagnetic spectrum, from the X-rays to the radio domain. In particular, BCGs residing in cool-core clusters, i.e. rich clusters with a relaxed morphology and showing a relevant drop (down to $10^{4} \mathrm{~K}$ ) in the X-ray temperature of the central $(r \lesssim 10-100 \mathrm{kpc}) \mathrm{ICM}$, are likely to host an active galactic nucleus (AGN) and have an intense on-going star formation activity. Optical nebulæ (e.g. Cowie et al. 1983; Cavagnolo et al. 2008) along with extended Ly $\alpha$ (e.g. $\mathrm{Hu}$ 1992) and far-ultraviolet continuum emissions (e.g. O'Dea et al. 2004; O'Dea et al. 2010) have been also detected around these BCGs. The co-existence of these phenomena and their tight relation with the properties of the galaxies environment (i.e. cool-core clusters) makes these BCGs a peculiar class of massive ellipticals, suggesting that they are the stage of very intricate baryon cycle processes. Different scenarios (e.g. AGN and/or star photoionisation, shocks, hot X-ray plasma penetrating cold gas) describing the origin and interplay between this phenomena, as well as the mechanisms regulating the BCGs AGN and star formation activity, have been proposed (e.g. Ferland et al. 2009; O'Dea et al. 2010). However, these scenarios are still a matter of debate, in particular for galaxies at intermediate-redshift where the spatial disentanglement between the different processes is limited by the resolving power of the instruments. In this regard, state-of-the-art integral-field unit instruments (e.g. MUSE) seem to give an answer to this problem, opening a new window on the description of galaxies inhabiting the far Universe.

In this context, we inquire the stellar and gas properties (i.e. kinematics, stellar mass, star formation rate) of the radio-loud (e.g. Kale et al. 2015) brightest cluster galaxy inhabiting the cool-core cluster (e.g. Rizza et al. 1998) Abell 2667 (see Fig. 1, left panel), a rich class-3 cluster, located in the southern celestial hemisphere at redshift $z=0.2343$.

\section{Data analysis and results}

To unveil the complex dynamical structure of the BCG, we exploit the exquisite quality of the data from the ESO IFU instrument MUSE at VLT. MUSE observations of the Abell 2667 cluster were taken under the GTO program 094.A-0115(A) (P.I. J. Richard) for a total exposure time of $2 \mathrm{~h}(4 \times 1800 \mathrm{~s})$ and a seeing of $<1^{\prime \prime}$ (FWHM). The rawdata are reduced by means of the ESO-MUSE pipeline (Weilbacher et al. 2014), and the Zurich Atmosphere Purge (ZAP, Soto et al. 2016).

We spatially resolve the BCG stellar kinematics with maps of stellar velocity and velocity dispersion by means of pPXF (Cappellari \& Emsellem 2004) while the estimate of 

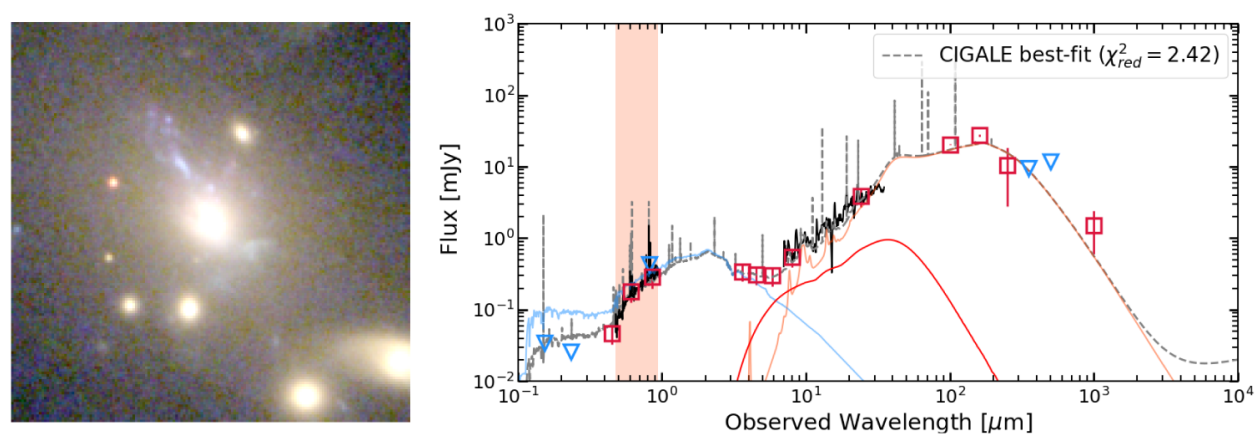

Figure 1. Left panel: HST RGB image $(\mathrm{F} 450 \mathrm{~W}+\mathrm{F} 606 \mathrm{~W}+\mathrm{F} 814 \mathrm{~W})$ of the BCG in Abell 2667 (the cutout size is $12.8^{\prime \prime} \times 12.8^{\prime \prime}$, i.e. $\sim 50 \mathrm{kpc} \times 50 \mathrm{kpc}$, centered at RA(J2000.0) $=$ $\left.23^{h} 51^{m} 39.37^{s} ; \operatorname{Dec}(J 2000.0)=-26^{\circ} 05^{\prime} 2.7^{\prime \prime}\right)$. Right Panel: CIGALE best-fit SED (gray dashedline) of the BCG photometric data (red open squares) and their $3 \sigma$ error. The orange and cyan solid lines show the contribution of the unattenuated stellar populations and dust, respectively; while the red solid line highlights the AGN torus emission. In black, the MUSE (vertical shaded area $4750-9350 \AA$ ) and IRS spectra of the galaxy are presented too.

the galaxy total stellar mass, stellar mass assembly history and its current star formation are obtained from MUSE spectra thanks to SINOPSIS (Fritz et al. 2017).

In agreement with what typically observed in massive ellipticals, our results indicate that the BCG is a dispersionally supported system with no sign of a coherent stellar velocity pattern (e.g. rotation) and with a spatially flat velocity dispersion $\left(\sigma_{0} \sim 216 \mathrm{~km} \mathrm{~s}^{-1}\right)$. From the relation by Zubovas \& King (2012) bounding the mass of supermassive black holes $\left(\mathrm{M}_{S M B H}\right)$ to the central velocity dispersion of elliptical galaxies in clusters, we estimate $\mathrm{M}_{S M B H} \sim 3.8 \times 10^{9} \mathrm{M}_{\odot}$. According to the statistical analysis by Smolčić (2009) on the physical properties of AGNs, our estimate of $\mathrm{M}_{S M B H}$ suggests that the Abell 2667 BCG is a massive elliptical hosting a low-ionisation nuclear emission-line region (LINER).

We measure the galaxy total stellar mass $\dagger$ equal to $\mathrm{M}_{\star} \sim 1.38 \times 10^{11} \mathrm{M}_{\odot}$. According to the BCG mass assembly history, $\sim 60 \%$ of $\mathrm{M}_{\star}$ formed more than $5.6 \mathrm{Gyr}$ ago. The value of $M_{\star}$ is confirmed by the analysis of the galaxy integrated photometric properties we perform thanks to the spectral energy distribution (SED) fitting code CIGALE (Noll et al. 2009; Boquien et al. 2018), using multi-wavelength data from rest-frame UV to IR (see Fig. 1, right panel). The SED fit indicates the BCG is hosting an obscured Type 2 AGN (as also pointed out by the analysis of Chandra data by Yang et al. 2018) whose emission does not contribute to the galaxy overall optical emission. The CIGALE analysis allows us to estimate the BCG star formation activity equal to $\neq \sim 14.5 \mathrm{M}_{\odot} / \mathrm{yr}$, thus locating the galaxy firmly along the main-sequence of galaxies as defined by Speagle et al. 2014. The intense star formation activity of the BCG obtained from the SED fit is confirmed by the analysis of the emission lines in MUSE spectra. Specifically, MUSE detects the presence of a young stellar population (younger than $20 \mathrm{Myr}$ and accounting for $\sim 0.6 \%$ of $\mathrm{M}_{\star}$ ) all around the galaxy core (see Fig. 2, left panel). These young stars are distributed along a filamentary and clumpy structure that can be clearly observed in the HST data.

$\dagger$ The stellar mass we refer is composed both by stars that are still in the nuclear-burning phase, and remnants (e.g. white dwarfs, neutron stars and stellar black holes).

$\ddagger$ The SFR we present is obtained from the conversion of the galaxy infrared luminosity into obscured star formation rate via the standard Kennicutt relation (Kennicutt 1998) for a Chabrier IMF, and adding the contribution of the unobscured component as in Santini et al. (2009). 

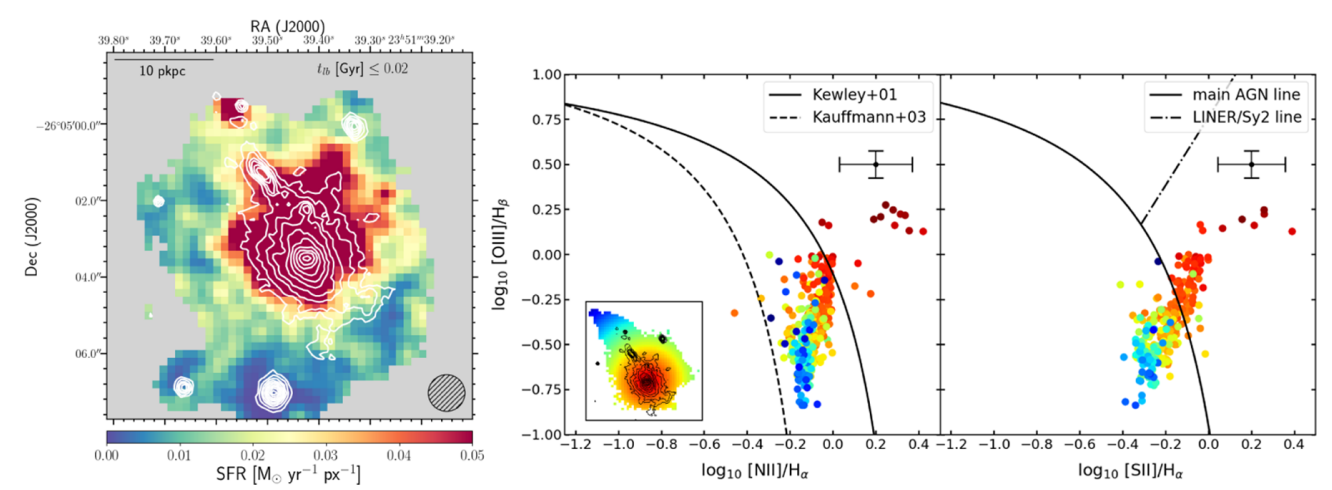

Figure 2. Left panel: SINOPSIS current SFR density map of the BCG with HST F450W contours superimposed. Right Panel: Spatially resolved BPT-[NII] (left) and BPT-[SII] (right) diagrams. Representative dots of the MUSE spaxels are colour-coded as a function of the projected spatial distance from the galaxy centre (see left bottom corner of the left diagram).

Since the Abell 2667 BCG has been proved to host an AGN, the results on the galaxy star formation rate obtained from emission lines have to be investigated in more detail as AGNs can contribute significantly to the gas ionisation and therefore to the total flux of the emission lines. To spatially disentangle the regions where the lines are emitted as a consequence of star formation than AGN, we resort to the empirical spectral diagnostic diagrams by Baldwin et al. (1981), the so-called BPTs, (see Fig. 2, right panel). According to BPTs, the very core of the BCG is consistent with a LINER while the gas in the galaxy outer regions seems to be ionised by processes that are compatible with both AGN activity and SF processes.

Ultimately, by studying the emission lines (specifically, $\mathrm{H}_{\beta}$ and [OIII] $\lambda 5007$ ) spatial distribution in different velocity channels (e.g. McNamara et al. 2014), we detect two extended and kinematically decoupled gas components in the velocity range $\Delta v_{\text {gas }}=$ $[100 ; 500] \mathrm{km} \mathrm{s}^{-1}$ and $\Delta v_{\text {gas }}=[-500 ;-100] \mathrm{km} \mathrm{s}^{-1}$, respectively.

\section{Discussion}

From our analysis, Abell 2667 seems to be a clear example of how intricate the baryon cycle is in the centre of massive galaxy clusters. Similarly to the results obtained by Tremblay et al. (2018) for the nearby Abell 2597 cool-core cluster, we might have caught Abell 2667 in the process of experiencing a baryon cycle where a shrink of star-forming giant clouds (i.e. the clumps observed in the HST data) made of cold ICM (e.g. Li \& Bryan 2014; Voit et al. 2015a) are accreting onto the BCG, refuelling it with gas thus turning on the central AGN and triggering its feedback (outflows). The combination of $H S T$ imaging and the detection of different velocity components of the ionised gas with respect to the rest-frame of the BCG, allow us to take into account also a scenario of galactic cannibalism. In this case, the clumps observed around the BCG could be stripped star-forming material left behind a small disc-galaxy (the major clump in the HST observations) that is merging along an inspiralling orbit and is undergoing disruption due to tidal interactions with the BCG and, possibly, ram-pressure stripping from the ICM. In this case, the galaxy AGN activity could be a pre-existent condition or could have been ignited as a consequence of accretion of material from the disc-galaxy.

\section{References}

Cowie, L. L., Hu, E. M., Jenkins, E. B., York, D. G., et al. 1983, ApJ, 272, 29

Cavagnolo, K. W., Donahue M., Voit, G. M., Sun M., et al. 2008, ApJ, 683, L107

Hu, E. M. 1992, ApJ, 391, 608 
O’Dea, C. P., Baum, S. A., Mack J., Koekemoer, A. M., Laor A., et al. 2004, ApJ, 612, 131

O'Dea, K. P., Quillen, A. C., O'Dea, C. P., Tremblay, G. R., Snios, B. T., Baum, S. A., Christiansen K., Noel-Storr J., et al. 2010, ApJ, 719, 1619

Ferland, G. J., Fabian, A. C., Hatch, N. A., Johnstone, R. M., Porter, R. L., van Hoof, P. A. M., Williams, R. J. R., et al. 2009, MNRAS, 392, 1475

Kale, R., Venturi, T., Cassano, R., Giacintucci, S., Bardelli, S., Dallacasa, D., Zucca, E., et al. 2015, A\& $A, 581, \mathrm{~A} 23$

Rizza, E., Burns, J. O., Ledlow, M. J., Owen, F. N., Voges, W., Bliton, M., et al. 1998, MNRAS, 301,328

Weilbacher, P. M., Streicher, O., Urrutia, T., Pécontal-Rousset, A., Jarno, A., Bacon, R., et al. 2014, ASPC, 485, 451

Soto, K. T., Lilly, S. J., Bacon, R., Richard, J., Conseil, S., et al. 2016, MNRAS, 458, 3210

Cappellari, M. \& Emsellem, E. 2004, PASP, 116, 138

Fritz, J., Poggianti, B. M., Bettoni, D., Cava, A., Couch, W. J., D’Onofrio, M., Dressler, A., Fasano G., et al. 2017, ApJ, 848, 132

Zubovas, K. \& King, A. R. 2012, MNRAS, 426, 2751

Smolčic̀, V. 2009, ApJ, 699, L43

Noll, S., Burgarella, D., Giovannoli, E., Buat, V., Marcillac, D., Muñoz-Mateos, J. C., et al. 2009, A\& $A, 507,1793$

Boquien, M., Burgarella, D., Roehlly, Y., Buat, V., Ciesla, L., Corre, D., Inoue, A. K., Salas, H., et al. 2018, arXiv e-prints

Yang, L., Tozzi, P., Yu, H., Lusso, E., Gaspari, M., Gilli, R., Nardini, E., Risaliti, G., et al. 2018, ApJ, 859, 65

Kennicutt, Jr. R. C. 1998, ARA\&A, 36, 189

Santini, P., Fontana, A., Grazian, A., Salimbeni, S., Fiore, F., Fontanot, F., Boutsia, K., Castellano M., et al. 2009, A\&\&A, 504, 751

Speagle, J. S., Steinhardt, C. L., Capak, P. L., Silverman, J. D., et al. 2014, ApJS, 214, 15

Baldwin, J. A., Phillips, M. M., Terlevich, R., et al. 1981, PASP, 93, 5

McNamara, B. R., Russell, H. R., Nulsen, P. E. J., Edge, A. C., Murray, N. W., Main, R. A., Vantyghem, A. N., Combes, F., et al. 2014, ApJ, 785, 44

Tremblay, G. R., Combes, F., Oonk, J. B. R., Russell, H. R., McDonald, M. A., Gaspari, M., Husemann, B., Nulsen, P. E. J., et al. 2018, ApJ, 865, 13

Li, Y. \& Bryan, G. L. 2014, ApJ, 789, 153

Voit, G. M., Donahue, M., Bryan, G. L., McDonald, M., et al. 2015a, Nature, 519, 203

\section{Discussion}

YUTAKA FuJITA: Could star formation be triggered by AGN activity?

IANI: AGN activity triggering star formation is definitely a possible/compatible scenario. However, we do not have enough scientific evidences to rule out shocks or merging.

Tomotsugu Goto: Can you explain the reason for the offset between the HST optical and MUSE data (i.e. $\mathrm{H}_{\beta}$, [OIII] $\left.\lambda 5007\right)$ ?

IANI: The small offset $\left(<0.2^{\prime \prime}\right)$ is due to a residual mismatch between HST and MUSE data coordinates. The mismatch has been corrected in our subsequent analysis.

Maud Galametz: How about the other objects in the field-of-view? Are they kinematically related to the BCG?

IANI: According to their redshift, the closest sources (on the sky-plane) to the BCG are galaxies inhabiting the very same cluster but we have not investigated their kinematics yet. 